\title{
Cardiac lymphoma with first manifestation of recurrent syncope-a case report and literature review
}

This article was published in the following Dove Press journal:

International Medical Case Reports Journal

5 February 2010

Number of times this article has been viewed

Joseph Nybo Lin

Johns Hopkins Bloomberg School of Public Health, Baltimore, MD, USA

\begin{abstract}
Cardiac involvement, as an initial presentation of malignant lymphoma is rare. We report a 42-year-old Taiwanese man with frequent syncope. Routine electrocardiography disclosed complete atrioventricular block. Before permanent pacemaker implantation was undertaken, transthoracic echocardiography revealed a solid mass infiltrating the left atrium and interatrial septum. Transesophageal echocardiography proved cardiac neoplasm with biatrial infiltration. Endomyocardial tissue biopsy was performed under angiography guide. Pathology diagnosis unraveled large B-cell lymphoma. Despite aggressive chemotherapy, the patient's condition worsened and he died 2 months later.
\end{abstract}

Keywords: primary cardiac lymphoma, complete atrioventricular block

\section{Introduction}

Cardiac lymphoma is a rare disease with a high mortality rate. When this malady is diagnosed prognosis is usually grim due to the advanced stage of myocardial involvement at presentation. It is defined as non-Hodgkin's lymphoma with heart and/or the pericardium involvement only. Primary cardiac lymphoma accounts for $1.3 \%$ of primary cardiac tumors and $0.5 \%$ of the extranodal lymphomas. ${ }^{1}$ However, disseminated cardiac lymphoma is more common, with an incident rate at $15 \%$ 30\%, of all lymphoma in various post-mortem series. ${ }^{2,3}$ The prognosis of both primary and disseminated cardiac lymphoma remains grave, largely because of late presentation and substantial delay at the critical point of initial diagnosis. Here, we report a uremic patient presenting with frequent syncope. His intriguing clinical course is delineated below.

\section{Case report}

A 42-year-old Taiwanese man with diabetic nephropathy in a uremic stage received regular hemodialysis in our hospital. He presented to our outpatient department with frequent syncope over a period of one month. Electrocardiogram revealed complete atrioventricular block with junctional escape rhythm (Figure 1). Before a permanent pacemaker was implanted, transthoracic echocardiography revealed a massive infiltrative thickening of the inferior aspect of the left atrial septum and interatrial septum mimic thrombus (Figure 2). Ensuing transesophageal echocardiography revealed a mass attached to the interatrial septum and protruding into both atrial cavities (Figure 3). Assayed tumor markers were all within the normal range (carcinoembryonic antigen [CEA], alpha-fetoprotein [AFP], cancer antigen [CA 19-9]). An epicardial permanent pacemaker implantation was performed due to the right atrial mass and fear of tumor
Correspondence: Joseph Nybo Lin Johns Hopkins Bloomberg School of Public Health, 615N.Wolfe Street,

Baltimore, MD 21205, USA

Tel +l 4432877277

Email josephsimion@yahoo.com.tw 


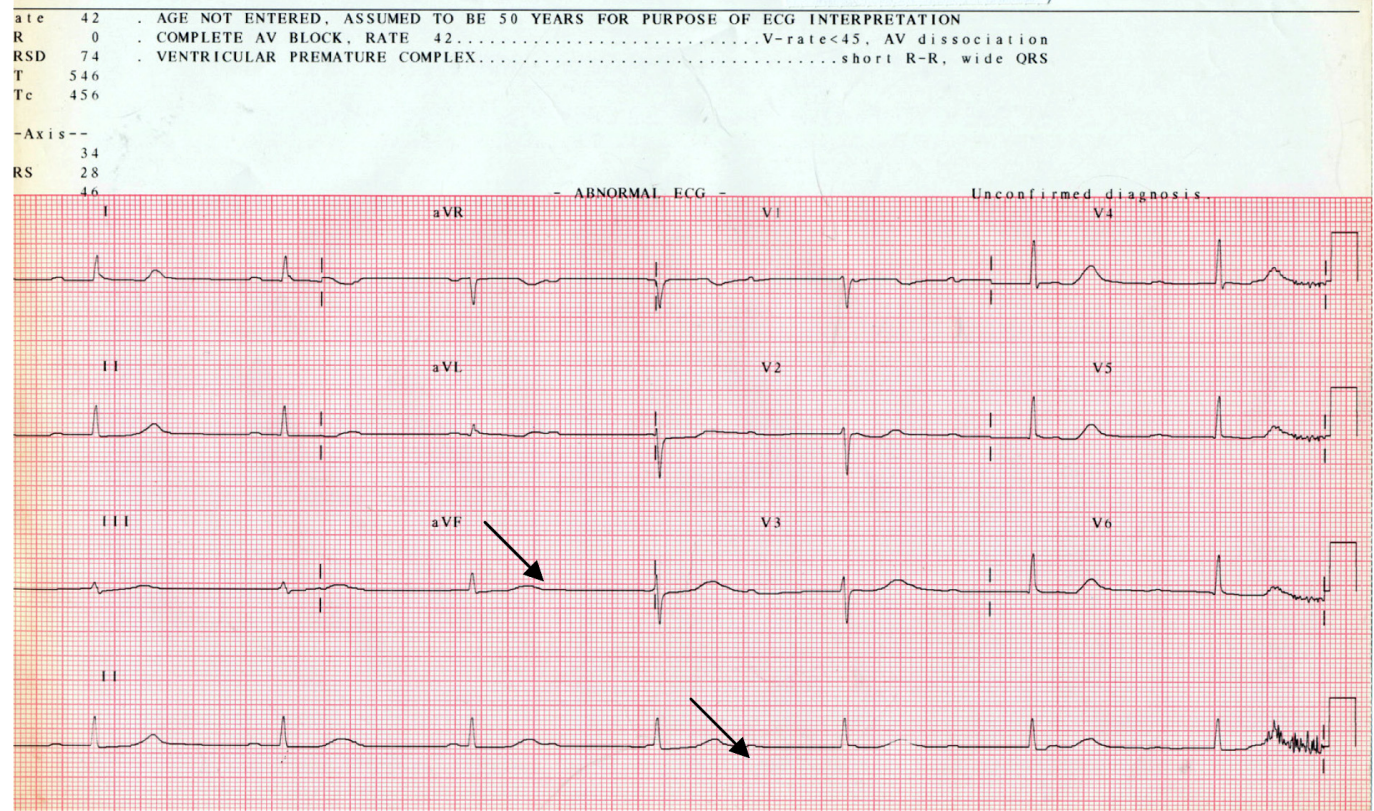

Figure I Electrocardiogram revealing complete atrioventricular block with junctional escape rhythm (arrows).

embolization. During the procedure of pacemaker implantation a pericardial biopsy was performed.

Multislice computed tomography (MSCT) revealed a bulky infiltrative mass $8.62 \mathrm{~cm} \times 6.45 \mathrm{~cm} \times 9 \mathrm{~cm}$ at the posterior-lateral and inferior aspect of the left atrium with invasion to myocardium, the interatrial septum, tricuspid valve, right atrium, pericardium, left hilar region and left pleura (Figure 4). There are lymph nodes in the paratracheal regions, paraaortic regions and highest mediastinum. To confirm the occult tumor origin and check if there were metastases, we arranged positron electric tomography (PET). After intravenous injection of $10 \mathrm{mCi}$ of $\mathrm{F}^{18}$-labeled 2-deoxyglucose (FDG), upper body and brain studies were performed with a Philips Gemini GXL PET/CT scanner. Images were reconstructed using line of response (LOR) reconstruction with computer tomography (CT) attenuation correlation.

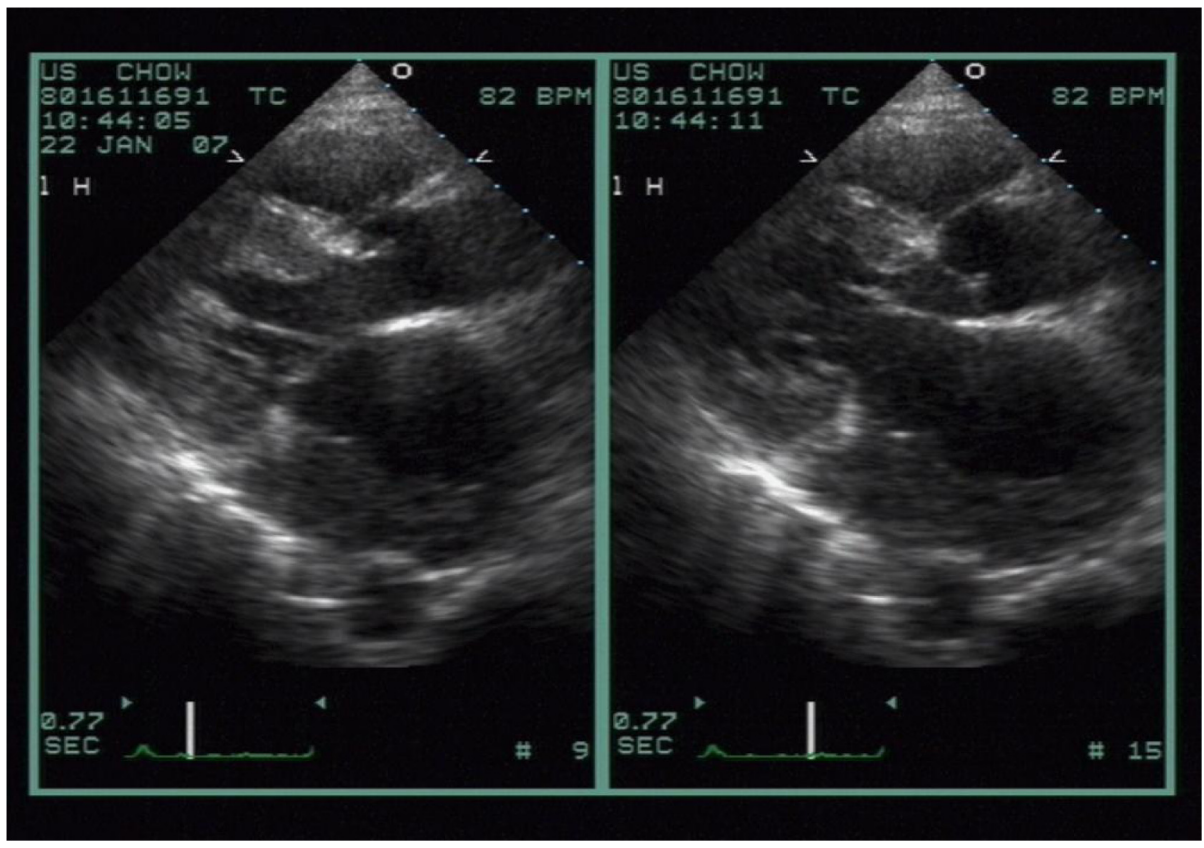

Figure 2 An ultrasonogram showing a massive infiltrative thickening of the posterior aspect of the left atrial septum and interatrial septum. 


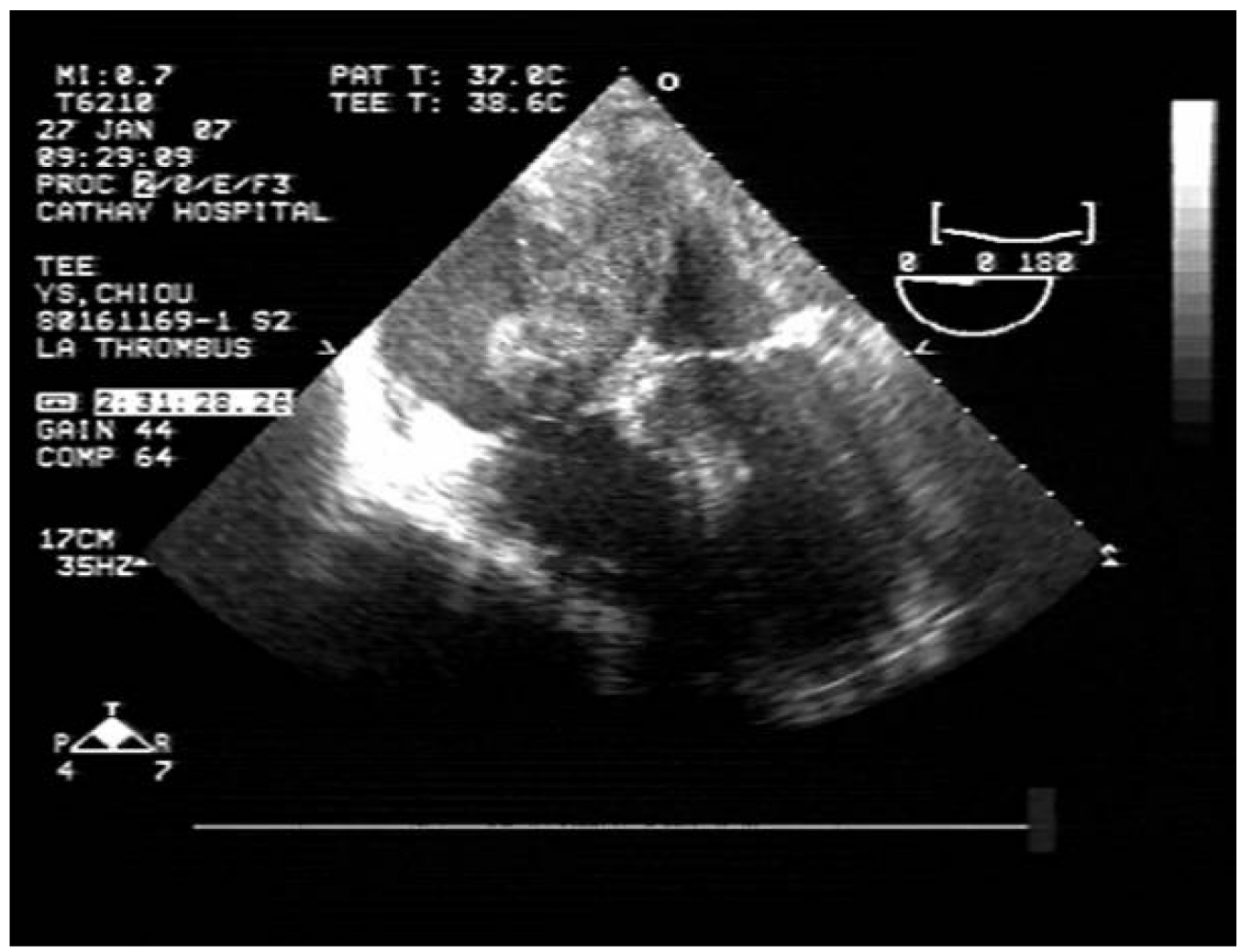

Figure 3 Transesophageal echocardiography showing tumor masses attached to the interatrial septum and protruding into both atrial cavities.

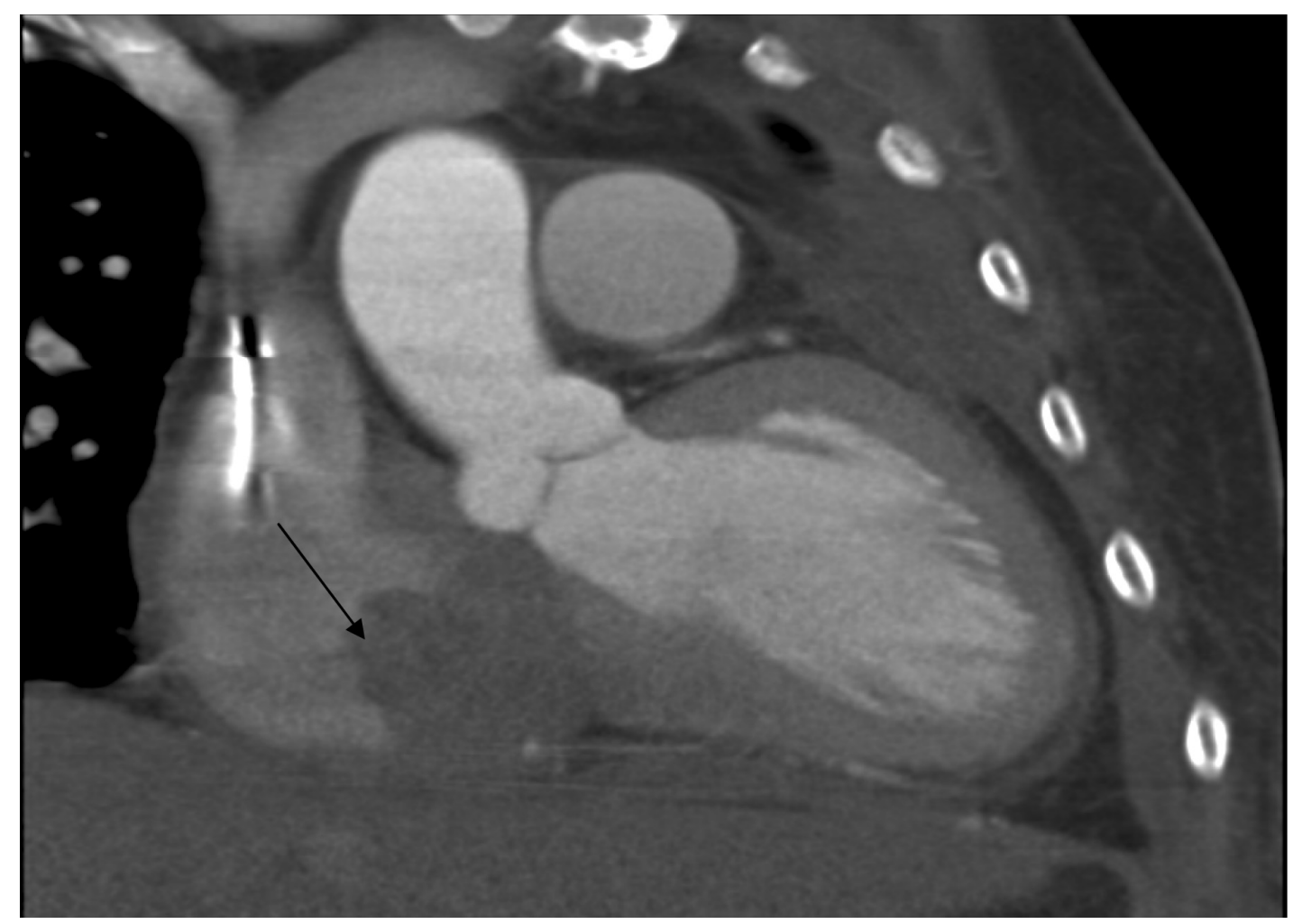

Figure 4 A multislice computed tomogram showing a bulky infiltrative mass about $8.62 \mathrm{~cm} \times 6.45 \mathrm{~cm} \times 9 \mathrm{~cm}$ at posterior-lateral and inferior aspect of the left atrium with invasion to myocardium, the interatrial septum, tricuspid valve, right atrium, pericardium, left hilar region, and left pleura (arrow). 
FDG-PET scans revealed increased glucose metabolism in the right atrium (RA), interatrial septum and left atrium (LA). The maximal standardized uptake value (SUV) value was 9.24. However, the result showed no abnormal radiotracer uptake in the pleura and lymph nodes (Figure 5).

Transvenous endomyocardial biopsy was undertaken, under fluoroscopic guidance, for pathologic diagnosis. The pathology report confirmed the presence of a diffuse large B-cell lymphoma. Immunohistochemically, the large tumor cells are strongly although diffusely positive for CLA (common leukocyte antigen) and CD20 (a marker for B-cells), admixed with small reactive CD3+/CD5+ T cells, but negative for cytokeratin, S-100, chromogranin, CD56, desmin, FLI-1, and CD34 (Figure 5). The patient was thus referred to a hematologist for chemotherapy with regimens of $\mathrm{CHOP}$ (cyclophosphamide, doxorubicin, vincristine, and prednisone) for 3 courses, with rituximab for 2 courses. Before the first course, a bone marrow biopsy was performed and the pathology report showed no evidence of bone marrow involvement. One month after chemotherapy, noncapture of the permanent pacemaker was found during hemodialysis with symptoms of malaise. The permanent pacemaker was thus adjusted by increasing output to maintain capture. Unfortunately, the patient suffered sudden cardiac death 2 months after chemotherapy.

\section{Discussion}

Cardiac tumors can be divided into primary cardiac tumors from heart and secondary cardiac tumors metastasized to heart. The documented incidence of primary cardiac tumors ranged from $0.17 \%$ to $0.19 \%$ in unselected autopsy series. Approximately $75 \%$ of primary cardiac tumors are benign, with the residual $25 \%$ being malignant. Fifty percent of benign tumors are myxomas. Among cardiac malignant tumors: angiosarcomas; rhabdomyosarcomas; malignant mesotheliomas; and fibrosarcomas are among most common. Lymphomas may arise from the heart, although the occurrence is rare. Primary cardiac lymphoma accounts for just $1.3 \%$ of primary cardiac tumors and $0.5 \%$ of the extranodal lymphomas. ${ }^{14}$

Cardiac involvement at first presentation of malignant lymphoma is rare. When compared with cardiac lymphoma, disseminated cardiac lymphoma occurs more frequently. Cardiac lymphoma invades primarily the right atrium, followed by the right ventricle, the left ventricle, and finally the atrial septum. ${ }^{5,6}$ Primary cardiac lymphomas originating in left atrium, as found in this case are rare. This makes the differential diagnosis of myxomas or atrial thrombus important. Myxomas often involve the left atrium and might protrude into the left ventricle. ${ }^{7}$ Atrial thrombus is often found in the left atrial appendage under the condition of rheumatic mitral

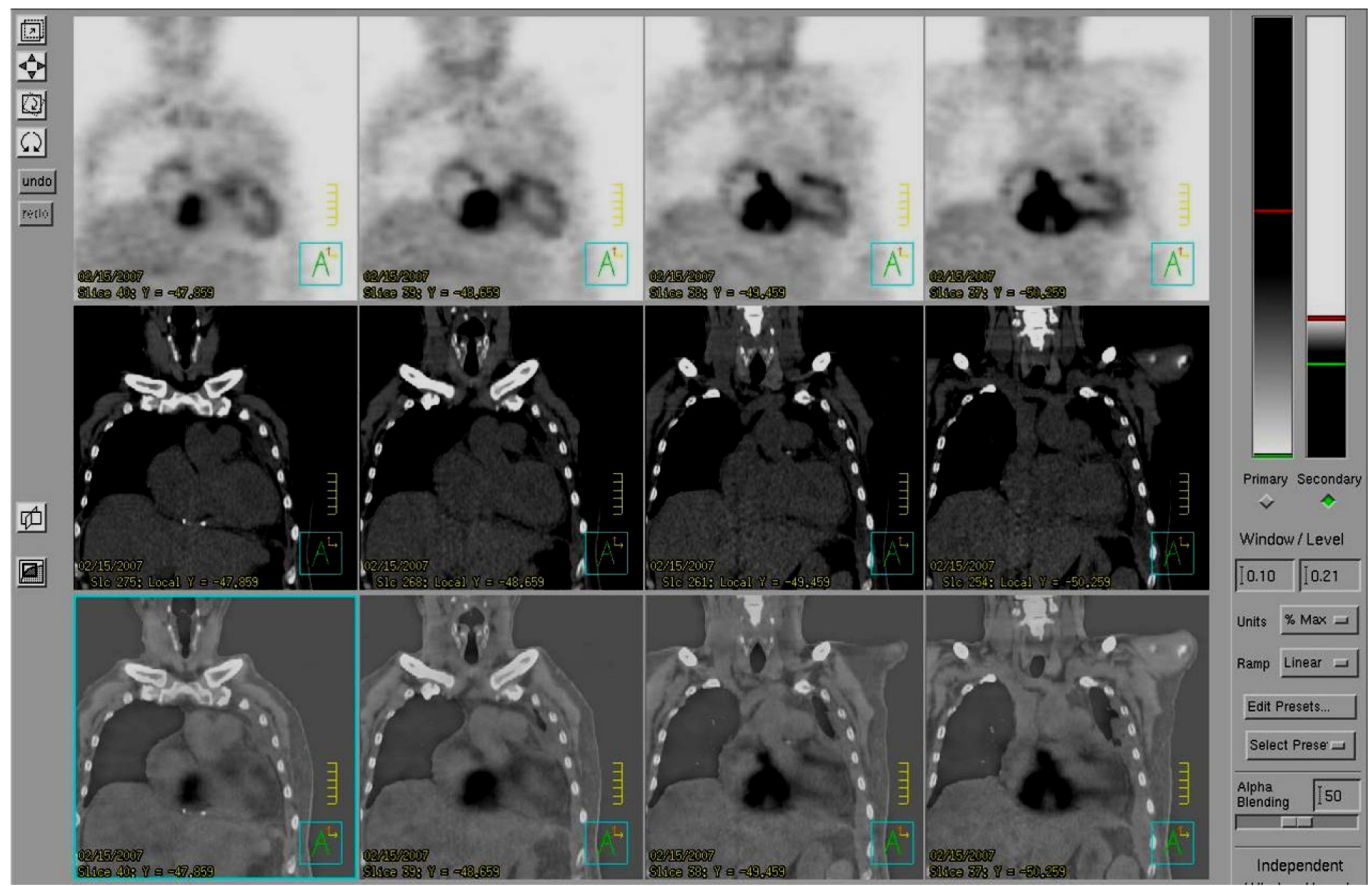

Figure 5 FDG-PET revealed remarkably increased glucose metabolism in the RA, interatrial septum, LA, and in a tumor mass protruding from the posterior aspect of pericardium.

Abbreviation: FDG-PET, $\mathrm{F}^{18}$-labeled 2-deoxyglucose positron electric tomography. 


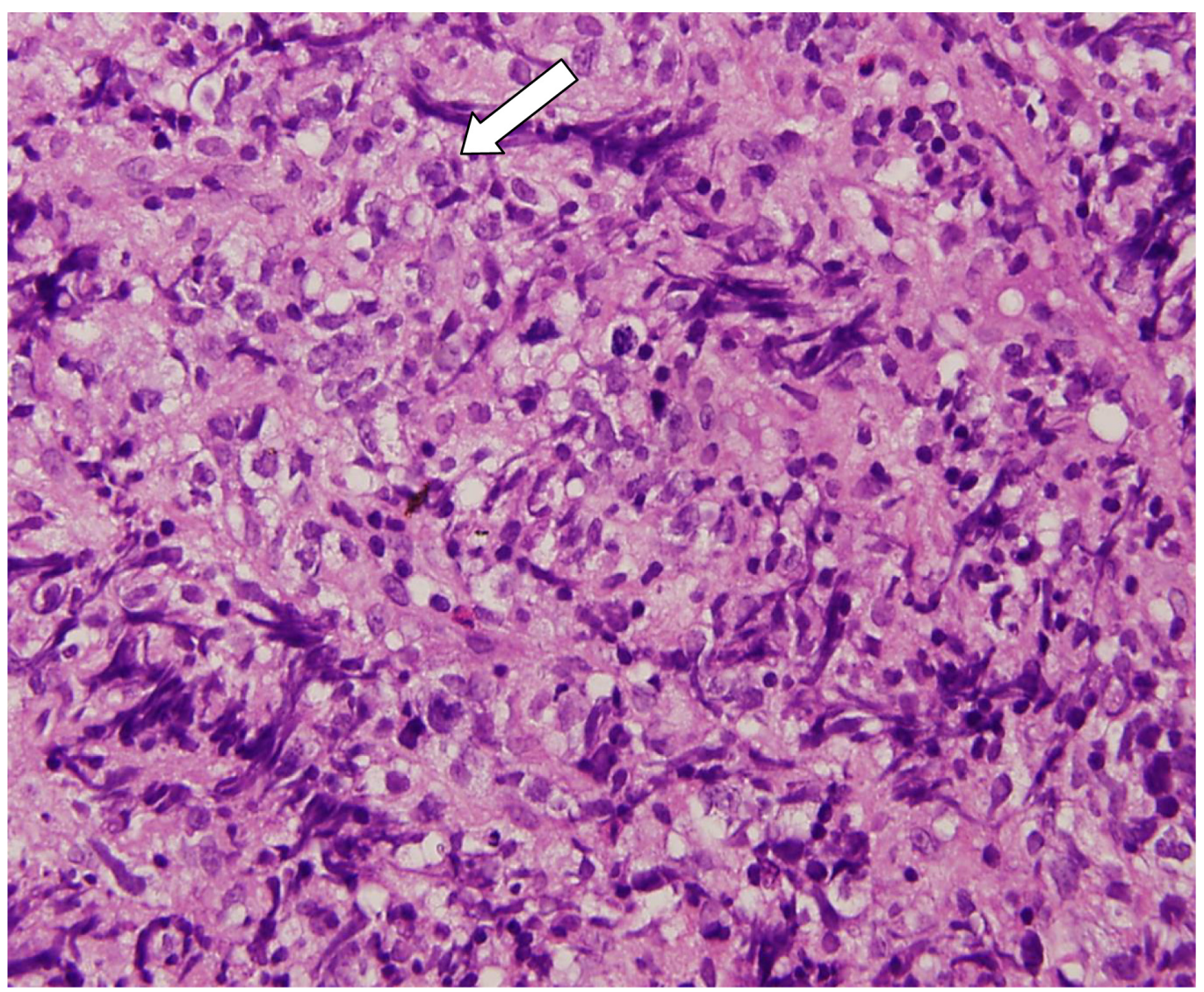

Figure 6 Section showing monotonous proliferation of large centroblast-like lymphoid cells having round to irregular nuclei, with vesicular chromatin and distinct I to 3 nucleoli and a moderate amount of cytoplasm (arrow).

stenosis or atrial fibrillation. Our patient presented with syncope and complete atrioventricular block. The reported age of patients with this condition, at presentation, is usually in the mid-60s although range is from 13- to 90 -year-olds. ${ }^{1}$ There is no pathognomonic clinical picture suggestive of primary cardiac lymphoma. All clinical presentations are related to the site of primary cardiac lymphoma involvement. Clinical presentations varies range from: congestive heart failure; dyspnea; ${ }^{8}$ fainting; syncope; atrial or ventricular arrhythmia; $;{ }^{911}$ neoplastic pulmonary embolism; ${ }^{12}$ pericardial effusion; ${ }^{13}$ pleural effusion; myocardial infarction; stroke; ${ }^{14}$ and sudden cardiac death. Elevation of the erythrocyte sedimentation rate, lactate dehydrogenase and inerleukin-2 receptor concentration are the main laboratory indicative features. ${ }^{15}$ Electrocardiography findings include: atrial flutter; fibrillation; bundle branch block and low voltage. ${ }^{9-11}$ Transthoracic echocardiography is a basic tool to identify pericardial effusion, pleural effusion and lesion of tumor. However, it does yield lower sensitivity. Additionally, transesophageal echocardiography provides better visualization of any suspicious cardiac mass and cardiac structure than transthoracic echocardiography. ${ }^{16}$
Computer tomography and magnetic resonance imaging (MRI) provide more precise assessment of tumor morphology: composition, number and extent than echocardiography. They are also superior in the detection of pericardium and paracardiac structures such as in the mediastinum, great vessels and lungs. ${ }^{17}$ Cardiac lymphoma with pericardium or pleura involvement could be diagnosed by cytology of associated pericardial or pleural effusion. If cytological diagnosis from serous fluid is inclusive, invasive biopsy should then be performed immediately. ${ }^{1}$ The prognosis of primary cardiac lymphoma could be improved with early diagnosis and early chemotherapy. Biopsy procedures such as: transesophageal echocardiography-guided biopsy, ${ }^{18}$ transvenous angiography-guided biopsy, ${ }^{1,7}$ mediastinoscopic biopsy or thoracoscopic biopsy are less invasive. Although direct thoracotomy biopsy is more invasive it yields a more accurate diagnosis rate. In pathology, the majority of primary cardiac lymphoma are large B-cell type, B-cell neoplasms. In immunophenotypic studies, cardiac lymphomas can be distinguished from other cardiac neoplasms on the basis of their reactivity for common leukocyte antigen and pan B-cell markers (CD 20) or pan T-cell makers (CD 3). Diffuse B-cell 
lymphoma accounts for about $80 \%$ of primary cardiac lymphomas in immunocompetent patients. In immunodeficiency patients, small noncleaved or immunoblastic lymphomas are more frequent. ${ }^{19}$ Increased glucose metabolism in the myocardium and/or pericardium, without abnormal exracardiac radiotracer uptake found by FDG-PET, would confirm the lymphoma being confined to the heart. Treatment includes chemotherapy, radiotherapy and surgery. Some researchers recommend a therapeutic strategy which includes CHOP chemotherapy. however, $\mathrm{CHOP}$ chemotherapy with rituximab is more effective than CHOP chemotherapy on its own Other strategies include chemotherapy with radiotherapy, which appear to prolong the survival period. ${ }^{1}$

In summary, primary cardiac lymphoma is a rare and often fatal malignancy with varied clinical presentation depending upon the site of tumor involvements. Early diagnosis and early treatment promises better prognoses. Transthoracic echocardiogram is traditionally used as a screening tool for any cardiac mass although it lacks the precise diagnostic rate of other techniques. Transesophageal echocardiogram, an invasive and operator dependent technique, provides better visualization of suspicious cardiac masses and cardiac structure, especially in though involving the atria. CT or MRI yields more detailed views of the mass location and extent of the tumor as compared to echocardiography. Biopsy for pathology diagnosis and therapy should be done as soon as possible after first presentation as the tumor growth rate is rapid and the diminishing prognosis is grave.

\section{Disclosures}

The authors report no conflicts of interest relevant to this study.

\section{References}

1. Ceresoli GL, Ferreri AJ, Bucci E, Ripa C, Ponzoni M, Villa E. Primary cardiac lymphoma in immunocompetent patients: diagnostic and therapeutic management. Cancer. 1997;80(8):1497-1506.

2. Mukai K, Shinkai T, Tominaga K, Shimosato Y. The incidence of secondary tumors of the heart and pericardium: a 10-year study. Jpn J Clin Oncol. 1988;18(3):195-201.
3. McDonnell PJ, Mann RB, Bulkley BH. Involvement of the heart by malignant lymphoma: a clinicopathologic study. Cancer. 1982;49(5):944-951.

4. McAllister HA, McAllister JJF. Tumors of the cardiovascular system. In: Atlas of Tumor Pathology. Washington DC: Armed Forces Institute of Pathology; 1978.

5. Grebenc ML, Rosado de Christenson ML, Burke AP, Green CE, Galvin JR. Primary cardiac and pericardial neoplasms: radiologicpathologic correlation. Radiographics. 2000;20(4):1073-1103.

6. Saotome M, Yoshitomi Y, Kojima S, Kuramochi M. Primary cardiac lymphoma-a case report. Angiology. 2002;53(2):239-241.

7. Nguyen DT, Meier CR, Schneider D. Primary cardiac lymphoma mimicking left atrial myxoma in an immunocompetent patient. $J$ Clin Oncol. 2008;26(1):150-152.

8. Roberts CS, Gottdiener JS, Roberts WC. Clinically undetected cardiac lymphoma causing fatal congestive heart failure. Am Heart $J$. 1990;120(5):1239-1242.

9. Hayes D, Liles DK, Sorrell VL. An unusual cause of new-onset atrial flutter: primary cardiac lymphoma. South Med J. 2003;96(8): 799-802.

10. Zaharia L, Gill PS. Primary cardiac lymphoma. Am J Clin Oncol. 1991;14(2):142-145.

11. Tai CJ, Wang WS, Chung MT, et al. Complete atrio-ventricular block as a major clinical presentation of the primary cardiac lymphoma: a case report. Jpn J Clin Oncol. 2001;31(5):217-220.

12. Bestetti RB, Soares FA, Soares EG, Oliveira JS. Primary lymphoma of the right atrium with fatal neoplastic pulmonary embolism. Am Heart $J$. 1992;124(4):1088-1090.

13. Rose AG, Grobbelaar J, Commerford PJ. Pericardial effusion and heart block caused by lymphoma. A case report. S Afr Med J. 1988.74(5): 240-242.

14. Binder J, Pfleger S, Schwarz S. Images in cardiovascular medicine. Right atrial primary cardiac lymphoma presenting with stroke. Circulation. 2004;110(17):e451-e452.

15. Makishima $\mathrm{H}$, Isobe $\mathrm{M}$, Imamura $\mathrm{H}$. A case of primary cardiac lymphoma: utility of serum soluble interleukin-2 receptor for noninvasive diagnosis. Int J Cardiol. 1998;65(3):291-293.

16. Mügge A, Daniel WG, Haverich A, Lichtlen PR. Diagnosis of noninfective cardiac mass lesions by two-dimensional echocardiography. Comparison of the transthoracic and transesophageal approaches. Circulation. 1991;83(1):70-78.

17. Dorsay TA, Ho VB, Rovira MJ, Armstrong MA, Brissette MD. Primary cardiac lymphoma: CT and MR findings. $J$ Comput Assist Tomogr. 1993;17(6):978-981.

18. Burling F, Devlin G, Heald S. Primary cardiac lymphoma diagnosed with transesophageal echocardiography-guided endomyocardial biopsy. Circulation. 2000;101(17):E179-E181.

19. Duong M, Dubois C, Buisson M, et al. Non-Hodgkin's lymphoma of the heart in patients infected with human immunodeficiency virus. Clin Cardiol. 1997; 20(5):497-502.
International Medical Case Reports Journal

\section{Publish your work in this journal}

The International Medical Case Reports Journal is an international, peer-reviewed open-access journal publishing original case reports from all medical specialties. Previously unpublished medical posters are also accepted relating to any area of clinical or preclinical science. Submissions should not normally exceed 2,000 words or

\section{Dovepress}

4 published pages including figures, diagrams and references. The manuscript management system is completely online and includes a very quick and fair peer-review system, which is all easy to use. Visit http://www.dovepress.com/testimonials.php to read real quotes from published authors. 